\title{
Avaliação do estado nutricional de atletas de ginástica olímpica do Rio de Janeiro e São Paulo
}

\author{
Nutritional status evaluation of olympic gymnastics athletes \\ from Rio de Janeiro and São Paulo, Brazil
}

Beatriz Gonçalves RIBEIRO'

Eliane de Abreu SOARES ${ }^{2}$

Com o objetivo de avaliar o estado nutricional de atletas de ginástica olímpica, do sexo feminino, foram estudadas 46 ginastas, de 11 a 14 anos, pertencentes a clubes e academias das cidades do Rio de Janeiro e São Paulo. O estudo antropométrico envolveu medidas de massa corporal, estatura, dobras cutâneas e perímetros. Observou-se diferença significativa para as dobras cutâneas tricipital, suprailíaca, da coxa e da panturrilha e para o percentual de gordura $(p<0,05)$. Para o estudo dietético foram utilizados recordatório de 24 horas e registro alimentar de três dias. As ginastas apresentaram baixo consumo energético. A percentagem de carboidratos, $50 \%$ a $58 \%$, e a ingestão protéica de 1,5 a 1,7 g/kg de peso mostraram-se baixa e adequada, respectivamente, frente ao proposto para atletas. O conteúdo de vitaminas foi satisfatório, com exceção do ácido fólico para as adolescentes cariocas. Os minerais julgados inadequados foram cálcio, magnésio, ferro e zinco. O estado nutricional de ferro não apresentou alterações.

Termos de indexação: avaliação nutricional, dieta, ferro, atletas, ginastas.

A B S TR A C T

The purpose of this study was to evaluate the nutritional status of 46 female olympic gymnastics athletes, aged 11 to 14 years old, attending private gymnastics clubs of the cities of Rio de Janeiro and São Paulo. The anthropometric assessment involved measurements of height, body mass, skinfold thickness and

\footnotetext{
1 Departamento de Nutrição e Dietética, Instituto de Nutrição, Universidade Federal do Rio de Janeiro. Centro de Ciências da Saúde - Bloco J, Cidade Universitária, Ilha do Fundão, 21941-590, Rio de Janeiro, RJ, Brasil. Correspondência para/ Correspondence to: B.G. RIBEIRO.E-mail: beatriz@cruiser.com.br

2 Departamento de Nutrição e Dietética, Instituto de Nutrição, Universidade Federal do Rio de Janeiro e da Universidade do Estado do Rio de Janeiro.
} 
circumferences. Significant difference was observed for the triceps, suprailiac, mid thigh and medial calf skinfolds and for the percentage of body fat $(p<0.05)$. For the assessment of dietary intake a 24-hour recall and a 3-day self-register were used. The energy consumption and the percentage of carbohydrates $(50 \%$ to $58 \%$ ) in the diet were low and the protein ingestion (1.5 to $1.7 \mathrm{~g} / \mathrm{kg}$ of body weight) was appropriate, according to values proposed for athletes. Vitamin intake was satisfactory, except for folic acid of gymnasts from Rio de Janeiro. From the minerals assessed, the intake of calcium, magnesium, zinc and iron was inadequate. No clinical signs of iron deficiency were observed.

Index terms: nutrition assessment, diet, iron, athletes, gymnasts.

\section{NTRODUÇ Ã O}

O número de mulheres praticantes de algum tipo de modalidade esportiva tem crescido consideravelmente. O aumento do esforço físico, decorrente do exercício, e a inadequação dietética expõem-nas a distúrbios orgânicos de todas as ordens. A literatura relata extensamente problemas relacionados a atletas femininas; amenorréia, anemia, osteoporose e distúrbios alimentares estão entre as principais disfunções que acometem este grupo, as quais parecem prevalecer mais em esportistas de elite (Webster \& Barr, 1995; O'Connor et al., 1996; Daly et al., 2000; Nickols-Richardson et al., 2000; Smolak et al., 2000).

A gênese destes distúrbios ainda é controversa. Conseqüentemente, muitas investigações são conduzidas focalizando-se uma multiplicidade de fatores, entre os quais os mais importantes são: baixo consumo energético, intenso treinamento físico, baixo percentual de gordura, alteração do perfil endócrino, ansiedade e estresse emocional (Committe... 1989; Wolman, 1990; Claessens et al., 1999; Deutz et al., 2000; Zetaruk, 2000).

As atletas de ginástica olímpica, para manter o peso corporal baixo e proporcional à altura requerido por este esporte, restringem o consumo alimentar (Benardot et al., 1989; Weimann et al., 2000). A redução do consumo energético pode provocar um atraso na puberdade, causar distúrbios no crescimento e induzir a amenorréia, a qual é um fator prejudicial ao desenvolvimento ósseo (Peltenburg et al., 1984; Weimann et al., 2000).

Estas atletas são mais baixas, magras e musculosas do que as praticantes de outras modalidades esportivas (Peltenburg et al., 1984; Haywood et al., 1986; Webster \& Barr, 1993; Daly et al., 2000) e do que adolescentes não atletas (Sinning \& Lindberg, 1972; Smit, 1973; Claessens et al., 1999). Se estas características são uma conseqüência do treinamento, de déficits nutricionais ou do somatório destes fatores, somente com base em futuros trabalhos estas hipóteses poderão ser aceitas ou refutadas.

Pesquisas com relação ao perfil hematológico de esportistas, especialmente análises de ferro plasmático, normalmente são conduzidas com esportes de resistência. Dados referentes a outras modalidades esportivas ainda são escassos. A deficiência de ferro em atletas varia de $9,5 \%$ a $57,0 \%$ para estados subclínicos e de $6,7 \%$ a $11,0 \%$ para anemia instalada, dependendo do grupo, idade e esporte (Raunikar \& Sabio, 1992; Constantini et al., 2000). A anemia, independente da sua origem, pode diminuir o consumo máximo de oxigênio, reduzir a capacidade de trabalho físico, diminuir a resistência e aumentar o risco de fadiga muscular (Rowland \& Kelleher, 1989; Constantini et al., 2000)

Diante do exposto e com base no fato de que o estado nutricional de atletas brasileiras é pouco documentado, surge o interesse em avaliar esta condição em praticantes femininas de ginástica olímpica, das cidades do Rio de Janeiro 
e São Paulo, através de informações sobre o consumo alimentar, composição corporal e perfil bioquímico. Os resultados desta pesquisa auxiliarão a traçar as características de um grupo de ginastas brasileiras, permitindo uma orientação nutricional direcionada a esta modalidade esportiva e a prevenção de agravos à saúde e ao rendimento físico.

\section{CASUISTICAE MÉTODOS}

Participaram do estudo 46 atletas de ginástica olímpica, do sexo feminino, de 11 a 14 anos, pertencentes a clubes e academias das cidades do Rio de Janeiro (10 do Clube Regatas do Flamengo, 5 do Clube Vasco da Gama, 2 da Academia Akxe) e São Paulo (8 da Associação Atlética Banco do Brasil, 10 do Esporte Clube Pinheiro, 11 da Academia Yashe). Foi incorporada ao modelo de análise a variável procedência, uma vez que este fator está associado a hábitos e consumo alimentar diferenciados. Após exposto o trabalho aos treinadores e às atletas, estas foram selecionadas através de uma análise probabilística aleatória. As ginastas treinavam em média 5 horas diárias, divididas em dois períodos (manhã e tarde), durante seis dias da semana.

A análise do consumo alimentar foi realizada utilizando-se dois métodos: um inquérito recordatório de 24 horas (R24) e um registro alimentar de três dias (R3). A coleta de dados foi efetuada pelas pesquisadoras, todas com formação na área de Nutrição.

O inquérito R24 referiu-se ao consumo nas 24 horas anteriores à entrevista. A fim de aumentar a confiabilidade dos dados e facilitar o preenchimento do R3, utilizou-se um Kit de utensílios, constituído por colheres de arroz, sopa, sobremesa, chá e café, concha média e copos de $240 \mathrm{~mL}$ e $165 \mathrm{~mL}$, todos de uso comum.

O registro alimentar incluiu três dias consecutivos, sendo excluído o fim de semana, e foi preenchido pelas atletas após orientação. A exclusão de um dia de final de semana justificou- -se pela participação delas em competições nesta ocasião, o que dificultaria as anotações, conforme relato pessoal das próprias ginastas.

Para o cômputo de energia e nutrientes ingeridos no R24 e R3, as medidas caseiras foram transformadas em gramas e mililitros (Soares et al., 1991; Pinheiro et al., 1993). O consumo alimentar foi analisado pelo Sistema de Avaliação Nutricional do Centro de Informática em Saúde da Escola Paulista de Medicina (Escola Paulista..., 1993) e comparado com as recomendações nutricionais do National Research Council (NRC) (National Research..., 1989). Em adição, calcularam-se os percentuais de energia oriundos dos carboidratos, proteínas e lipídios, bem como o total de gramas consumidos por $\mathrm{kg}$ de peso, sendo comparados com as orientações correntes para atletas (American Dietetic...,1993).

Através do registro alimentar bem como do recordatório de 24 horas, foi possível verificar quais eram as refeições realizadas com maior freqüência pelas adolescentes.

Foram tomadas medidas da massa corporal, estatura, dobras cutâneas e circunferências. As medições foram realizadas sempre antes do treinamento, no período da tarde, por um único avaliador experiente da área de educação física.

Para a medida de estatura, utilizou-se uma fita métrica com precisão de até décimos de centímetros $(\mathrm{mm})$, afixada na parede, sem rodapé, em cada clube e academia participante, estando a atleta em posição ortostática, com os pés juntos.

O peso corpóreo foi obtido empregando-se uma balança médica Filizola, com precisão de até 100 gramas, estando as atletas descalças e com o mínimo de roupa possível.

Para a avaliação das dobras cutâneas tricipital, bicipital, subescapular, suprailíaca, abdominal, da coxa e da panturrilha, utilizou-se um plicômetro Cescorf. Os perímetros (braço, coxa, perna) foram aferidos através de fita métrica com precisão de até décimos de centímetros $(\mathrm{mm})$. Estas medidas foram feitas do lado direito 
do corpo, sendo realizadas três mensurações alternadas em cada local e o valor médio utilizado como escore final (McArdle et al., 1992).

O percentual de gordura foi calculado segundo Slaughter et al. (1988). Ainda foram determinados o peso de gordura, o peso magro (McArdle et al., 1992) e o Índice de Massa Corporal (IMC), expresso em $\mathrm{kg} / \mathrm{m}^{2}$ (World..., 1985).

As atletas compareceram ao laboratório pela manhã, em jejum de 8 horas, para avaliação do estado nutricional de ferro. Foram determinados hemograma completo, ferro total, ferritina sérica e grau de saturação de transferrina. As concentrações de ferritina foram medidas através de radioimunoensaio, usando-se um Kit Diagnostic Products, USA. O ferro sérico e a capacidade de saturação da transferrina foram medidos usando o método colorimétrico automatizado (Kit Merck Brazi). O hematócrito foi analisado por um processo ultramicro (processo capilar convencional) e a hemoglobina foi estimada pelo método colorimétrico de cianometahemoglobina (Kit Bioclin do Brazil).

Considerou-se deficiência de ferro a existência, simultaneamente, dos seguintes critérios: ferritina sérica $<12(\mathrm{ng} / \mathrm{dL})$ e percentual de saturação de transferrina $<16 \%$. Em adição, quando as concentrações de hemoglobina encontravam-se abaixo de $120 \mathrm{~g} / \mathrm{L}$, as atletas eram classificadas como anêmicas (Rowland \& Kelleher, 1989).

Foram calculadas médias e desvios-padrão, considerando-se a procedência: Rio de Janeiro (RJ) e São Paulo (SP). As médias da idade, das variáveis antropométricas e do consumo alimentar foram avaliadas através do teste $t$ de Student. Para a verificação da variação do consumo médio de energia, macronutrientes e micronutrientes contidos no recordatório de 24 horas e nos três

Tabela 1. Médias e desvios-padrão para idade e para indicadores antropométricos das atletas de ginástica olímpica, segundo a procedência.

\begin{tabular}{|c|c|c|}
\hline \multirow[b]{2}{*}{ Variáveis } & \multicolumn{2}{|c|}{ Procedência } \\
\hline & $\begin{array}{c}\mathrm{R} \\
(\mathrm{n}=17)\end{array}$ & $\begin{array}{c}\text { SP } \\
(n=29)\end{array}$ \\
\hline Idade (anos) & $12,5 \pm 1,3$ & $13,0 \pm 1,0$ \\
\hline Estatura $(\mathrm{cm})$ & $147,4 \pm 0,1$ & $143,4 \pm 0,1$ \\
\hline Massa Corporal (kg) & $39,7 \pm 7,5$ & $37,3 \pm 6,7$ \\
\hline \multirow[t]{2}{*}{$\mathrm{IMC}^{1}$} & $18,1 \pm 2,2$ & $17,6 \pm 3,0$ \\
\hline & \multicolumn{2}{|c|}{ Dobras Cutâneas } \\
\hline Tricipital (mm) & $8,5 \pm 2,6$ & $4,3 \pm 1,8^{*}$ \\
\hline Bicipital (mm) & $4,8 \pm 1,6$ & $6,0 \pm 2,7$ \\
\hline Subescapular (mm) & $6,6 \pm 1,9$ & $5,6 \pm 1,3$ \\
\hline Suprailíaca (mm) & $7,8 \pm 2,9$ & $5,7 \pm 2,1^{*}$ \\
\hline Abdominal (mm) & $6,5 \pm 2,7$ & $6,0 \pm 2,6$ \\
\hline Coxa $(\mathrm{mm})$ & $13,6 \pm 4,8$ & $10,8 \pm 5,2^{*}$ \\
\hline Panturrilha (mm) & $10,2 \pm 3,8$ & $7,4 \pm 2,5^{*}$ \\
\hline$\%$ Gordura $^{2}$ & $16,5 \pm 3,6$ & $12,2 \pm 2,1$ * \\
\hline Peso Gordura $(\mathrm{kg})^{3}$ & $6,7 \pm 2,4$ & $6,5 \pm 2,2$ \\
\hline \multirow[t]{2}{*}{ Peso Magro $(\mathrm{kg})^{3}$} & $32,9 \pm 5,5$ & $30,8 \pm 5,0$ \\
\hline & \multicolumn{2}{|c|}{ Circunferências } \\
\hline $\operatorname{Braço}(\mathrm{cm})$ & $22,9 \pm 2,2$ & $22,9 \pm 2,4$ \\
\hline $\operatorname{Coxa}(\mathrm{cm})$ & $46,4 \pm 4,4$ & $43,5 \pm 6,2$ \\
\hline Panturrilha (cm) & $29,3 \pm 4,1$ & $29,6 \pm 2,2$ \\
\hline
\end{tabular}

(1) IMC = Índice de Massa Corporal = massa $(\mathrm{kg}) / \mathrm{estatura}\left(\mathrm{m}^{2}\right) ;{ }^{(2)} \% \mathrm{G}=$ percentual de gordura; ${ }^{(3)}$ Peso calculado em função do \% de gordura; ${ }^{(*)} \mathrm{p}<0,05$ associado ao teste " $\mathrm{t}$ ", segundo procedência. 
registros alimentares, foi aplicado o teste Análise de Variância (ANOVA). Em ambos os procedimentos adotou-se um nível de significância de $5 \%(p<0,05)$. Estas análises foram realizadas utilizando-se o programa estatístico MINITAB, versão 7.2 (Minitab...,1989).

\section{RESULTADOS}

A idade média das atletas paulistas foi maior que a das cariocas. Por outro lado, o percentual médio de gordura destas foi significantemente mais elevado que o daquelas $(p<0,05)$. A média das dobras cutâneas tricipital, suprailíaca, da coxa e da panturrilha foi significativamente maior nas ginastas do Rio de Janeiro $(p<0,05)$. Para o IMC não observou-se diferença entre os grupos (Tabela 1).

A análise comparativa da ingestão alimentar entre os três registros (auto-avaliação) e recordatório de 24 horas não mostrou diferença significante entre os resultados das quatro anotações. Portanto, assumiu-se os dados do recordatório de 24 horas, permitindo-se estimar as médias de consumo de energia, macronutrientes, vitaminas e minerais (Tabela 2).

O consumo energético foi maior entre as ginastas cariocas (1521 kcal) em comparação com as paulistas (1423 kcal), mas ambos os valores se situaram abaixo dos preconizados para adolescentes não atletas. $\mathrm{A}$ ingestão média de carboidratos foi significantemente maior nas ginastas cariocas $(p<0,05)$. De acordo com os dados obtidos, as atletas de São Paulo consumiram maior quantidade de proteínas e lipídios do que as do Rio de Janeiro (Tabela 2). O percentual médio de energia fornecida pelos macronutrientes foi diferente entre os grupos (Figura 1).

De um modo geral, observando-se a ingestão média de vitaminas e minerais, as ginastas paulistas consumiram quantidades superiores às das cariocas. Verificou-se que o consumo de

Tabela 2. Médias e desvios-padrão do consumo de energia e nutrientes das ginastas estimado pela média dos dados do recordatório de 24 horas, segundo a procedência.

\begin{tabular}{|c|c|c|c|}
\hline Variáveis & $\begin{array}{c}\text { RJ } \\
(n=17)\end{array}$ & & $\begin{array}{c}\text { SP } \\
(n=29)\end{array}$ \\
\hline Energia (kcal) & $1521 \pm 446,9$ & & $1423 \pm 345,7$ \\
\hline Proteínas $(\mathrm{g} / \mathrm{d})^{1}$ & $61,1 \pm 22,6$ & & $67,4 \pm 23,2$ \\
\hline $\mathrm{g} / \mathrm{kg} / \mathrm{d}^{2}$ & $1,5 \pm 0,5$ & & $1,7 \pm 0,7$ \\
\hline Carboidratos (g/d) & $235,6 \pm 74,4$ & & $179,2 \pm 54,7^{*}$ \\
\hline $\mathrm{g} / \mathrm{kg} / \mathrm{d}$ & $5,9 \pm 1,8$ & & 4,8 \\
\hline Lipídios (g/d) & $53,8 \pm 25,8$ & & $54,5 \pm 23,4$ \\
\hline \multirow[t]{2}{*}{$\mathrm{g} / \mathrm{kg} / \mathrm{d}$} & $1,4 \pm 0,6$ & & $1,5 \pm 0,6$ \\
\hline & & Vitaminas & \\
\hline Vitamina C (mg) & $79,1 \pm 89,5$ & & $196,2 \pm 101,5^{*}$ \\
\hline Tiamina(mg) & $1,2 \pm 0,4$ & & $1,2 \pm \quad 0,4$ \\
\hline Riboflavina (mg) & $1,2 \pm 0,5$ & & $1,4 \pm 0,5$ \\
\hline Niacina (mg) & $17,2 \pm 8,7$ & & $20,3 \pm 8,3$ \\
\hline Vitamina B6 (mg) & $1,4 \pm 0,7$ & & $1,3 \pm 0,6$ \\
\hline Folato $(\mu \mathrm{g})$ & $120,1 \pm 61,4$ & & $160,9 \pm 66,9 *$ \\
\hline \multirow[t]{2}{*}{ Vitamina E (mg) } & $5,0 \pm 4,0$ & & $5,1 \pm 4,4$ \\
\hline & & Minerais & \\
\hline Cálcio (mg) & $578,9 \pm 274,9$ & & $546,8 \pm 260,3$ \\
\hline Magnésio(mg) & $102,6 \pm 54,1$ & & $159,1 \pm 48,8^{*}$ \\
\hline Ferro (mg) & $11,3 \pm 4,2$ & & $11,3 \pm 3,3$ \\
\hline Zinco (mg) & $4,6 \pm 2,8$ & & $6,4 \pm$ \\
\hline
\end{tabular}

${ }^{(1)} \mathrm{g} / \mathrm{d}=$ gramas por dia; ${ }^{(2)} \mathrm{g} / \mathrm{kg} / \mathrm{d}$ = gramas por kg de massa corporal por dia; ${ }^{(*)} \mathrm{p}<0,05$ associado ao teste " $\mathrm{t}$ ", segundo procedência. 

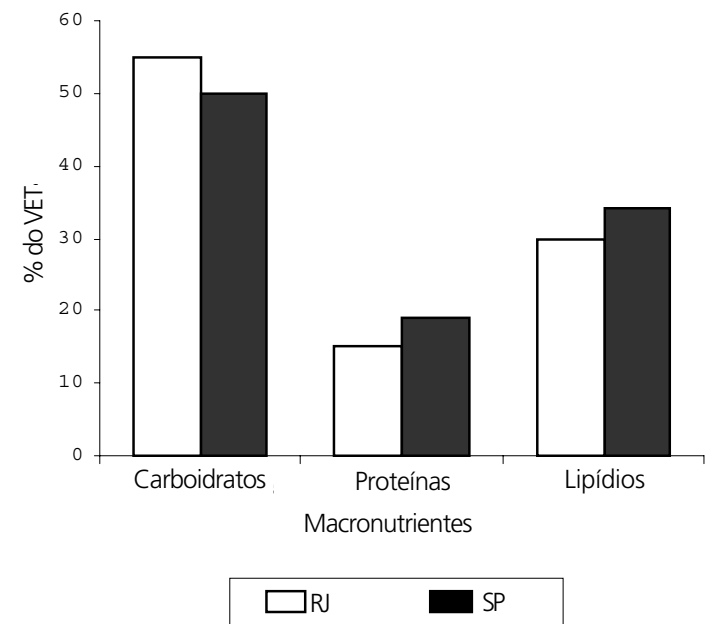

Figura 1. Distribuição percentual dos macronutrientes, em função do valor energético total, estimado pela média do recordatório de 24 horas, segundo a procedência.

vitamina $C$ e magnésio foi significantemente maior entre as paulistas $(p<0,05)$ e o consumo de cálcio foi maior entre as cariocas (Tabela 2), embora no caso deste último nutriente o valor tenha sido muito abaixo das recomendações nutricionais (National Research..., 1989).

Mais de $50 \%$ das atletas realizavam diariamente quatro refeições: desjejum, almoço, lanche e jantar. A ceia foi a refeição menos referenciada por ambos os grupos estudados (Figura 2).
Quanto ao estado nutricional de ferro, os parâmetros avaliados (hemoglobina, hematócrito, ferro sérico, saturação de transferrina e ferritina sérica) demonstraram que as ginastas apresentaram valores médios superiores aos pontos de corte propostos para designar deficiência de ferro e anemia (Tabela 3).

\section{DISCUSSÃO}

A estatura e o peso corporal vêm sendo considerados duas desvantagens em atletas que praticam ginástica olímpica, cujos exercícios requerem agilidade, leveza e força. No entanto, a própria natureza do esporte é vista com um fator seletivo por si só: crianças mais baixas e aquelas com um peso corporal menor têm maior impulsão e saltam levemente (Claessens et al., 1999; Zetaruk, 2000).

Pesquisas confirmam um perfil antropométrico e de composição corporal diferenciado nestas atletas. Entretanto, os autores discutem se estas características são decorrentes do treinamento ou de déficits nutricionais (Sinning \& Lindberg, 1972; Bernink et al., 1983; Benardot \& Czerwinski, 1991; Claessens et al., 1999).

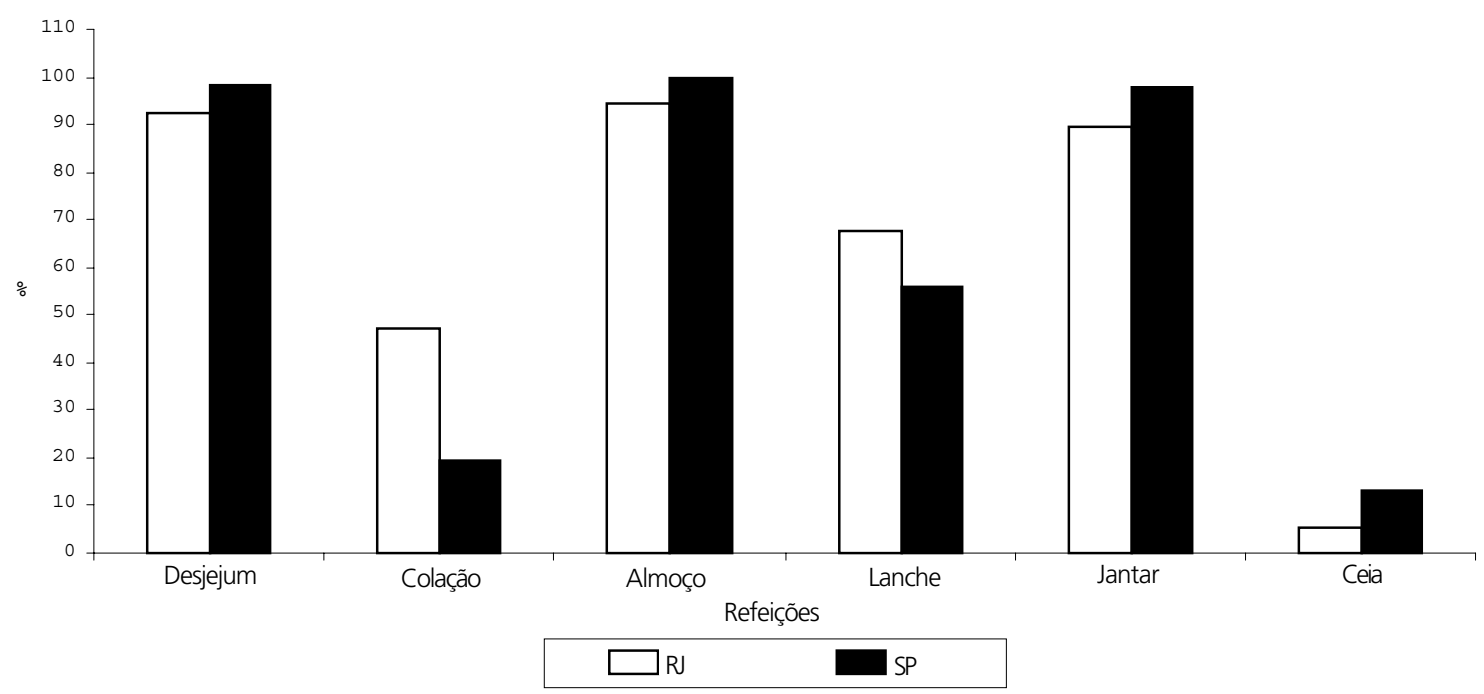

Figura 2. Distribuição percentual das refeições realizadas pelas atletas, obtida a partir do inquérito dietético, segundo a procedência. 
Tabela 3. Médias e desvios-padrão das características hematológicas das atletas, segundo a procedência.

\begin{tabular}{lcc}
\hline & \multicolumn{2}{c}{ Procedência } \\
\cline { 2 - 3 } Indicadores & $\mathrm{R}$ & $\mathrm{SP}$ \\
& $(\mathrm{n}=17)$ & $136,0 \pm 10,0$ \\
& $138,0 \pm 12,0$ & $40,0 \pm 0,3$ \\
Hemoglobina $(\mathrm{g} / \mathrm{L})$ & $42,0 \pm 0,1$ & $64,2 \pm 7,3$ \\
Hematócrito(\%) & $66,3 \pm 6,8$ & $29,0 \pm 11,5$ \\
Ferro sérico $(\mu \mathrm{g} / \mathrm{dl})$ & $32,0 \pm 12,6$ & $62,6 \pm 25,0$ \\
Saturação de transferrina(\%) & $78,5 \pm 31,0$ & \\
Ferritina sérica $(\mathrm{ng} / \mathrm{mL})$ &
\end{tabular}

A estatura das atletas de ginástica olímpica estudadas mostrou-se diferente daquela de adolescentes brasileiras da mesma idade - Instituto Nacional de Alimentação e Nutrição (INAN) (Instituto Nacional..., 1990). As ginastas são mais baixas confirmando ser este perfil característico de jovens praticantes desta modalidade esportiva.

A multiplicidade de técnicas utilizadas para avaliar o percentual de gordura corporal em ginastas não nos permitiu fazer comparações das adolescentes estudadas com as atletas de outros países. Contudo, ressalta-se que, para atletas de 11 a 14 anos, Theintz et al. (1993) encontraram $14,9 \%$ de gordura corporal, percentual similar aos nossos resultados, enquanto Benardot \& Czerwinski (1991) obtiveram 9,2\% nas atletas da Associação Independente de Clubes de Ginastas dos EUA, valor abaixo do presente estudo.

Guedes (1994), analisando o perfil corporal de adolescentes brasileiras, residentes em Londrina (Paraná), encontrou $21,79 \%$ de gordura corporal, número acima dos observados nas ginastas desta pesquisa. Os nossos achados sugerem que a intensa participação em atividades físicas modifica o conteúdo de gordura corporal, gerando valores mais baixos para as jovens atletas em comparação com os encontrados em adolescentes brasileiras não atletas.

Especialmente para ginastas femininas e bailarinas, o consumo de energia é freqüentemente descrito como baixo, em relação à idade e ao peso corporal, quando comparado às recomendações nutricionais (Deutz et al., 2000). Estas atletas, normalmente, limitam o consumo energético para reduzir o peso corporal, tendo em vista uma melhoria no desempenho profissional (Benardot et al., 1989; Claessens et al., 2000). Deutz et al. (2000), avaliando o balanço energético em 42 ginastas, identificaram um déficit de $743 \mathrm{kcal}, \mathrm{e}$, segundo eles, o estilo de vida atlético associado ao baixo consumo energético pode facilmente induzir problemas relacionados com a nutrição, os quais são incompatíveis com a saúde e o ótimo rendimento.

Os consumos energéticos médios das atletas de ginástica olímpica foram de $1521 \mathrm{kcal}$ (cariocas) e $1423 \mathrm{kcal}$ (paulistas) (Tabela 2). A recomendação energética para adolescentes femininas de 11 a 14 anos é de $2200 \mathrm{kcal} / \mathrm{dia}$ (National Research..., 1989) - 679 kcal e 777 kcal a menos para as ginastas cariocas e paulistas, respectivamente. Apesar de se considerar a existência de uma grande variação intra-individual no gasto energético, estes valores merecem uma análise cuidadosa quando desvios no consumo de energia trazem consigo alterações metabólicas e fisiológicas, como diminuição da taxa metabólica de repouso, redução da secreção de insulina (Organización..., 1991) e menor atividade de 5 monodiiodinase, enzima envolvida no metabolismo da tireóide (Burger et al.,1987). Além disso, o balanço energético negativo está associado à incidência de amenorréia entre as atletas (American College..., 1997). No presente estudo não foi levado em consideração o gasto energético despendido na atividade física específica, o que, para as ginastas estudadas, pressupõe um déficit energético maior.

De acordo com o Dietary Guidelines for American e a Canadian Nutrition Recomendations 
188 | B.G. RIBEIRO \& E.A. SOARES

(American Dietetic..., 1993), a proporção adequada de macronutrientes para atletas é de $60 \%$ a $65 \%$ de carboidratos não mais do que $30 \%$ do total energético de lipídios e 1,5 g/ $\mathrm{kg}$ do peso corporal/dia de proteínas.

O estudo dietético de ambos os grupos de ginastas demonstrou uma variação percentual de $50 \%$ a $58 \%$ no consumo energético oriundo dos carboidratos (Figura 1). Este percentual pode ser considerado baixo, quando o grupo de referência é formado por atletas. Sherman (1995) recomenda um consumo diário de 8 a $11 \mathrm{~g}$ de carboidrato/kg de peso para quem treina intensamente durante duas horas ou mais. Considerando que as ginastas treinavam aproximadamente $2 \mathrm{~h} 30 \mathrm{~min} / \mathrm{sessão}$ (duas vezes/dia), os consumos de $5,9 \mathrm{~g} / \mathrm{kg}$ de peso (cariocas) e $4,8 \mathrm{~g} / \mathrm{kg}$ de peso (paulistas) representam significativamente, em termos biológicos, bem menos do que o esperado (Tabela 2). Esta baixa ingestão de carboidratos associada ao reduzido consumo energético poderá contribuir para uma redução nos estoques de glicogênio muscular, resultando em estresse adicional ao organismo e prejuízos tanto nos treinamentos como em competições.

Conforme sugerem pesquisas, a quantidade necessária de proteína para ativar uma máxima deposição no músculo é de $1,5 \mathrm{~g} / \mathrm{kg}$ de peso corporal/dia e o fator limitante para esta síntese ocorrer é o consumo energético e não o protéico (American Dietetic..., 1993). Segundo Tarnopolsky (1993), a ingestão de proteínas acima de $1,76 \mathrm{~g} / \mathrm{kg} / \mathrm{dia}$ é oxidada como energia e não é notado aumento na síntese protéica.

A ingestão protéica das ginastas participantes do nosso estudo variou de 15\% a 19\% do consumo energético diário (Figura 1). Apesar de estes valores estarem adequados (Tabela 2) em relação às recomendações estabelecidas para adolescentes de 1,0 g/kg de peso corporal/dia (National Research..., 1989) e às sugeridas para atletas de 1,5 a 2,0 g/ $\mathrm{kg} / \mathrm{dia}$ (American Dietetic..., 1993), a baixa ingestão energética constatada nas atletas estudadas indica uma possível utilização protéica para fins energéticos, com riscos relacionados ao crescimento.

Lopez-Varela et al. (2000) verificaram entre ginastas adolescentes do Canadá um mesmo perfil de comportamento alimentar, representado por um baixo consumo energético e de carboidratos associado a um aumento da ingestão protéica e lipídica.

Observou-se que a dieta das atletas de ambas as procedências, com respeito ao conteúdo de vitaminas, mostrou-se satisfatória, com exceção do ácido fólico para as cariocas (Tabela 2).

Os minerais julgados inadequados foram cálcio, magnésio, ferro e zinco. A inadequação do consumo do magnésio e zinco deve ser vista com cuidado, pois os dados de composição destes nutrientes ainda não estão disponíveis para todos os alimentos. Além disso, a recomendação nutricional (National Research..., 1989) é um parâmetro de referência do consumo de nutrientes para a população, e uma ingestão abaixo dos valores propostos denota um risco na conduta alimentar. No entanto, a ingestão deficiente destes nutrientes vem sendo bem documentada em outras pesquisas (Moffatt, 1984; Hickson et al., 1986; Loosli et al., 1986; Kirchner et al., 1995; Lindholm et al., 1995).

A ingestão de cálcio mostrou-se insatisfatória para ambos os grupos estando, aproximadamente $45 \%$ abaixo das recomendações. O consumo adequado deste nutriente é particularmente importante, visto que fraturas ocorrem mais comumente em atletas com baixa densidade óssea, irregularidades menstruais e ingestão insuficiente de cálcio (Düpe et al., 1997; Nickols-Richardson et al., 1999). Principalmente durante a infância e adolescência, a prevenção primária da osteoporose está relacionada à máxima obtenção da densidade mineral óssea através da ingestão adequada de cálcio (Vuori, 1996). Além disso, esta substância é também perdida no suor e o exercício pode aumentar a sua necessidade em atletas. 
Embora o consumo de ferro tenha sido considerado baixo, nenhuma de nossas ginastas foi classificada como anêmica. O percentual de saturação de transferrina e as concentrações de ferritina não demonstraram um estado de deficiência deste nutriente. Valores individuais de algumas atletas indicaram baixa concentração de ferro plasmático, mas os outros indicadores não confirmaram que elas apresentavam um estado clínico e subclínico de carência de ferro. Quanto a estas evidências, podemos sugerir um mecanismo compensatório do organismo, através de uma maior eficiência na absorção deste nutriente, devido a um baixo consumo alimentar.

\section{CONCLUSÃO}

No global as ginastas estudadas apresentaram um perfil antropométrico clássico, característico das atletas que praticam ginástica olímpica. Este modelo pode estar associado ao processo seletivo do esporte e à inadequação nutricional do grupo, apontada pelo presente trabalho e respaldada por outras pesquisas as quais reiteram esta associação.

Analisando o consumo alimentar dos grupos separadamente, pode-se concluir que, em termos quantitativos - ingestão energética e distribuição de macronutrientes, a dieta das atletas cariocas demonstrou um perfil mais adequado. No entanto, embora tenha sido evidenciado um menor consumo energético entre as ginastas paulistas, estas apresentaram um conteúdo maior de micronutrientes em suas dietas.

Com relação ao estado nutricional de ferro, as atletas não apresentaram alterações, apesar do comprometimento alimentar.

Sugere-se o planejamento de ações conjuntas para reforçar os conhecimentos em nutrição de técnicos, de pais, de responsáveis e principalmente das atletas, visando uma melhora na conduta alimentar, a fim de garantir que a nutrição não seja um fator limitante no rendimento e na saúde destas adolescentes.

\section{REFERENCIAS BIBLIOGRÁFICAS}

AMERICAN COLLEGE OF SPORTS MEDICINE. The female athlete triad. Medicine and Science in Sports and Exercise, Baltimore, v.29, n.5, p.1-9, 1997.

AMERICAN DIETETIC ASSOCIATION. Position of the American Dietetic Association and the Canadian Dietetic Association: nutrition for physical fitness and athletic performance for adults. Journal of the American Dietetic Association, Chicago, v.93, n.6, p.691-696, 1993.

BENARDOT, D., SCHWARZ, M., HELLER, D.W. Nutrient intake in young, highly competitive gymnast. Journal of the American Dietetic Association, Chicago, v.89, n.3, p.401-403, 1989.

BENARDOT, D., CZERWINSKI, C. Selected body composition and growth measures of junior elite gymnasts. Journal of the American Dietetic Association, Chicago, v.91, n.1, p.29-33, 1991.

BERNINK, M.J.E., ERICH, W.B.M., PELTENBURG, A.L., ZONDERLAND, M.L., HUISVELD, I.A. Height, body composition, biological maturation and training in relation to socio-economic status in girl gymnasts, swimmers, and controls. Growth, Bar Harbor ME, v.47, n.3, p.1-12, 1983.

BURGER, A., CONNEL, M., SCHEIDEGGER, K. Monode-iodination of triiodothyronine and reverse triiodothyronine during low and high calorie diets. Journal of Clinical Endocrinology and Metabolism, Baltimore, v.65, n.1, p.829-835, 1987.

CLAESSENS, A.L., LEFEVRE, J., BEUNEN, G., MALINA, R.M. The contribution of anthropometric characteristics to performance scores in elite female gymnasts. Journal of Sports Medicine and Physical Fitness, Torino, v.39, n.4, p.355-360, 1999.

COMMITTEE ON SPORTS MEDICINE. Amenorrhea in adolescent athletes. Pediatrics, Evanston IL, v.84, n.2, p.394-395, 1989.

CONSTANTINI, N.W., ELIAKIM, A., ZIGEL, L., YAARON, M., FALK, B. Iron status of highly active adolescents: evidence of depleted iron stores in gymnasts. International Journal Sports Nutrition Exercise and Metabolism, v.10, n.1, p.62-70, 2000. 
190 | B.G. RIBEIRO \& E.A. SOARES

DALY, R.M., RICH, P.A., KLEIN, R., BASS, S.L. Short stature in competitive prepubertal and early pubertal male gymnasts: the result of selection bias or intense training? Journal of Pediatrics, St. Louis MO, v.137, n.4, p.510-516, 2000

DEUTZ, R.C., BENARDOT, D., MARTIN, D.E., CODY, M.M. Relationship between energy deficits and body composition in elite female gymnasts and runners. Medicine and Science in Sports and Exercise, Baltimore, v.32, n.3, p.659-668, 2000.

DÜPPE, H., GARDSELL, P., JOHNELL, O., NILSSON, B.E., RINGSBERG, K. Bone mineral density, muscle strength and physical activity: a population based study of 332 subjects aged 15-42 years. Acta Orthopaedica Scandinavica, Copenhage, v.68, n.2, p.97-103, 1997.

ESCOLA PAULISTA DE MEDICINA. Centro de informática em saúde. Programa de apoio a nutrição CIS/EPM, [programa de computador]. Versão 2.5. São Paulo : Brasil, 1993.

GUEDES, D.P. Composição corporal: princípios, técnicas e aplicações. 2.ed. [S.I.] : Ceitec, 1994. 93p.

HAYWOOD, K.M., CLARCK, B.A., MAYHEW, J.L. Differential effects of age-group gymnastics and swimming on body composition, strength, and flexibility. International Journal of Sports Medicine, Stuttgart, v.26, n.1, p.416-420, 1986.

HICKSON, J.F., SCHRADER, J., TRISCHLER, L.C. Dietary intakes of female basketball and gymnastics athletes. Journal of the American Dietetic Association, Chicago, v.86, n.2, p.251-253, 1986.

INSTITUTO NACIONAL DE ALIMENTAÇÃO E NUTRIÇÃO. Pesquisa nacional sobre saúde e nutrição: perfil de crescimento da população brasileira de 0 a 25 anos. Brasília, 1990. 60p.

KIRCHNER, E.M., LEWIS, R.D., O'CONNOR, P.J. Bone mineral density and dietary intake of female college gymnasts. Medicine and Science in Sports and Exercise, Baltimore, v.27, n.2, p.543-549, 1995.

LINDHOLM, C., HAGENFELDT, K., HAGMAN, U. A nutrition study in juvenile elite gymnasts. Acta Paediatrics, Stockholm, v.84, n.1, p.273-277, 1995.
LOOSLI, A.R., BENSON, J., GILLIEN, D.M., BOURDET, K. Nutrition habits and knowledge in competitive adolescent female gymnasts. Physician and Sportsmedicine, v.14, n.8, p.118-130, 1986.

LOPEZ-VARELA, S., MONTERO, A., CHANDRA, R.K., MARCOS, A. Nutritional status of young female elite gymnasts. International Journal for Vitamin and Nutrition Research, Bern, v.70, n.4, p.185-190, 2000.

MCARDLE, W.D., KATCH, F.I., KATCH, V.L. Fisiologia do exercício: energia, nutrição e desempenho humano. Rio de Janeiro : Guanabara, 1992. 510p.

MINITAB. Programa de estatística. Versão 7.2. [S.I.] : Minitab Inc/State College, 1989.

MOFFATT, R.J. Dietary status of elite female high school gymnast: inadequacy of vitamin and mineral intake. Journal of the American Dietetic Association, Chicago, v.84, n.11, p.1361-1363, 1984.

NATIONAL RESEARCH COUNCIL. Recommended Dietary Allowances. 10.ed. Washington DC: National Academy Press, 1989. 284p.

NICKOLS-RICHARDSON, S.M., O'CONNOR, P.J., SHAPSES, S.A., LEWIS, R.D. Longitudinal bone mineral density changes in female child artistic gymnasts. Journal of Bone and Mineral Research, New York, v.14, n.6, p.994-1002, 1999.

NICKOLS-RICHARDSON, S.M., MODLESKY, C.M., O'CONNOR, P.J., LEWIS, R.D. Premenarcheal gymnasts possess higher bone mineral density than controls. Medicine and Science in Sports and Exercise, Baltimore, v.32, n.1, p.63-69, 2000.

O'CONNOR, P.J., LEWIS, R.D., BOYD, A. Health concerns of artistic women gymnasts. Sports Medicine, Auckland, v.21, n.5, 321-325, 1996.

ORGANIZACIÓN PANAMERICANA DE LA SALUD. Conocimientos actuales sobre nutrición. 6.ed. Washington DC, 1991. 628p.

PELTENBURG, A.L., ERICH, W.B.M., BERNINK, M.J.E., ZONDERLAND, M.L., HUISVELD, I.A. Biological maturation, body composition, and growth of female gymnasts and control groups of schoolgirls and girls swimmers, aged 8 to 14 years: a cross-sectional survey of 1064 girls. International Journal of Sports Medicine, Stuttgart, v.5, n.1, p.36-42, 1984. 
PINHEIRO, A.V.B., LACERDA, E.M.A., BENZECRY, E.A., GOMES, M.C.S., COSTA, V.M. Tabela para avaliação do consumo alimentar em medidas caseiras. Rio de Janeiro : [s.n.], 1993. 64p. (produção independente).

RAUNIKAR, R.A., SABIO, H. Anemia in the adolescent athlete. American Journal of Diseases of Child, Chicago, v.146, n.3, p.1201-1205, 1992.

ROWLAND, T.W., KELLEHER, J.F. Iron deficiency in athlete. American Journal of Diseases of Child, Chicago, v.143, n.1, p.197-200, 1989.

SHERMAN, W.M. Metabolism of sugar and physical performance. American Journal of Clinical Nutrition, Bethesda, v.62, p.S228-S241, 1995. Supplement.

SINNING, W.E., LINDBERG, G.D. Physical characteristics of college age women gymnasts. Research Quarterly, Washington DC, v.43, n.2, p.226-234, 1972.

SLAUGHTER, M.H., LOHMAN, T.G., BOILEAU, R.A., HORSWILL, C.A., STILLMAN, R.J., LOAN, V., BEMBEN, D.A. Skinfolds equations for estimation of body fatness in children and youth. Human Biology, Detroit MI, v.60, n.5, p.709-723, 1988.

SMIT, P.J. Anthropometric observations on South African gymnasts. South African Medical Journal, Capetown, v.47, n.1, p.480-485, 1973.

SMOLAK, L., MURNEN, S.K., RUBLE, A.E. Female athletes and eating problems: a meta-analysis. International Journal Eating Disorders, v.27, n.4, p.371-380, 2000.

SOARES, E.A., PORTELLA, E.S., ISHII, M. Relação de medidas caseiras de 320 alimentos e respectivas gramagens. São Paulo : Universidade de São Paulo, 1991. 33p. (Ceane - Série divulgação científica).

TARNOPOLSKY, M.A. Protein, caffeine, and sports: guidelines for active people. Physician and Sportsmedicine, v.21, n.3, p.137-149, 1993.
THEINTZ, G.E., HOWAL, D.H., WEISS, U., SIZONENKO, P.C. Evidence for a reduction of growth potential in adolescent female gymnasts. Journal of Pediatrics, St. Louis MO, v.122, n.1, p.306-313, 1993.

VUORI, I. Peak bone mass and physical activity: a short review. Nutrition Reviews, New York, v.54, n.4, p.11-14, 1996

WEBSTER, B.L., BARR, S.I. Body composition analysis of female adolescent athletes: comparing six regression equations. Medicine and Science in Sports and Exercise, Baltimore, v.25, n.5, p.648-653, 1993.

WEBSTER, B.L., BARR, S.I. Calcium intakes of adolescent female gymnasts and speed skaters: lack of association with dieting behavior. International Journal of Sports Nutrition, v.5, n.1, p.2-12, 1995.

WEIMANN, E., WITZEL, C., SCHWIDERGALL, S., BOHLES, H.J. Peripubertal perturbations in elite gymnasts caused by sport specific training regimes and inadequate nutritional intake. International Journal of Sports Medicine, Stuttgart, v.21, n.3, p.210-215, 2000.

WOLMAN, R.L. Bone mineral density levels in elite female athletes. Annals of the Rheumatic Diseases, London, v.49, n.3, p.1013-1016, 1990.

WORLD HEALTH ORGANIZATION. Energy and protein requirements. Geneve, 1985. 221p. (Technical Report Series, 24).

ZETARUK, M.N. The young gymnast. Clinics in Sports Medicine, Philadelphia, v.19, n.4, p.757-780, 2000.

Recebido para publicação em 13 de dezembro de 2000 e aceito em 26 de maio de 2001. 